\title{
Preparing TEM Specimens and Atom Probe Tips by Laser Machining
}

\author{
Boris Rottwinkel1*, André Kreutzer ${ }^{1}$, Henry Spott ${ }^{1}$, Michael Krause ${ }^{2}$, Georg Schusser², \\ and Thomas Höche ${ }^{2}$ \\ 13D-Micromac AG, Technologie-Campus 8, 09126 Chemnitz, Germany \\ ${ }^{2}$ Fraunhofer Institute for Microstructure of Materials and Systems IMWS, Walter-Huelse-Strasse 1, 06120 Halle, Germany \\ *Rottwinkel@3d-micromac.com
}

\begin{abstract}
Preparation of electron-transparent thin specimens can be costly in terms of time and is often challenging. Materials and products are becoming more complex, and device components are getting smaller each year. On the other hand, analysis and diagnostic methods become more exacting. Lack of time and high costs for diagnostics force companies to speed up, simplify, and customize the analysis process. Ultra-short-pulsed laser-based sample preparation can speed up the process and make possible new sample geometries. This article shows the advantages of this technology and how it can be used to prepare TEM lamellas (H-Bar) and multiple APT tips or pillars.
\end{abstract}

Keywords: TEM lamellas, ultra-short-pulsed laser, laser ablation, APT tips, heating effects

\section{Introduction}

Over the past fifty years, lasers have found many applications in science and technology. The most important features of lasers are that photons are inherently free of elemental contamination, extremely high-energy densities can be focused to small diameters, and the laser beam can be precisely positioned using deflection mirrors. By reducing pulse lengths from a few nanoseconds down to the picosecond or femtosecond range, superficial surface heat damage from ablation can be reduced to depths of a few microns or less. Available for many decades, lasers have become extremely reliable processing tools in many areas, including the automotive industry (welding, drilling, and measuring), thin-film processing (scribing, patterning), and even esthetic surgery (dermabrasive ablation, LASIK).

In view of the outstanding characteristics of lasers as tools for micro machining, it is surprising that instruments for specimen preparation, for state-of-the-art analytical techniques, have not made use of laser technology. 3D-Micromac company has developed a dedicated laser micromachining instrument for specimen preparation. The name of this instrument indicates its purpose: microPREP (microscopy preparation) (Figure 1).

At sufficiently high power (or more precisely fluence), laser radiation is able to ablate all kinds of materials. Using ultra-short pulses and high pulse energies, ablation-based tools can also process materials that are transparent to the laser's wavelength. The ablation rate of laser micromachining is about six orders of magnitude larger than that of the Ga-ion beam used in focused ion beam (FIB) workstations, and it is even about four orders of magnitude higher than the milling rate of the Xe-plasma-FIB. Moreover, lasers can be very precisely positioned on a given workpiece and straightforwardly focused using standard optical elements. Since laser radiation consists just of photons (rather than an ion beam, which may have unwanted implantation effects), laser micro-machining is clean in terms of contamination. This article shows how this technology can be used to produce thin lamellae for transmission electron microscopy (TEM) and sharp tips for atom probe tomography (APT) [1-3].

\section{Materials Methods}

Tasks for the laser. Specimen preparation techniques need to be fast, reliable, cost-effective, and artifact-free on the micrometer scale or smaller. Traditional mechanical specimen preparation and FIB systems currently dominate the field. While the former is accompanied by the high costs of timeconsuming processes, the latter is characterized by the high costs of instrument ownership.

There are a number of specific capabilities of a laser-based thin-specimen preparation tool. Based on several patents [e.g., $4,5]$, the tool described here has a range of basic functionalities, including laser cutting from the bulk, followed by local laser thinning in an almost entirely automated fashion. Making use of a rugged, ultra-short-pulsed laser source, the process is characterized by low running costs, suitability for all materials of modern microelectronics, freedom from contamination, and a precision of about one micrometer.

Instrumental setup and performance. The main components of the microPREP instrument are a $532 \mathrm{~nm}$ laser source with $3 \mathrm{~W}$ average power and an objective lens focusing the laser beam to a small probe (beam diameter $\sim 10 \mu \mathrm{m}$ ). The pulse energy of up to $60 \mu \mathrm{J}$ enables high ablation rates compared with

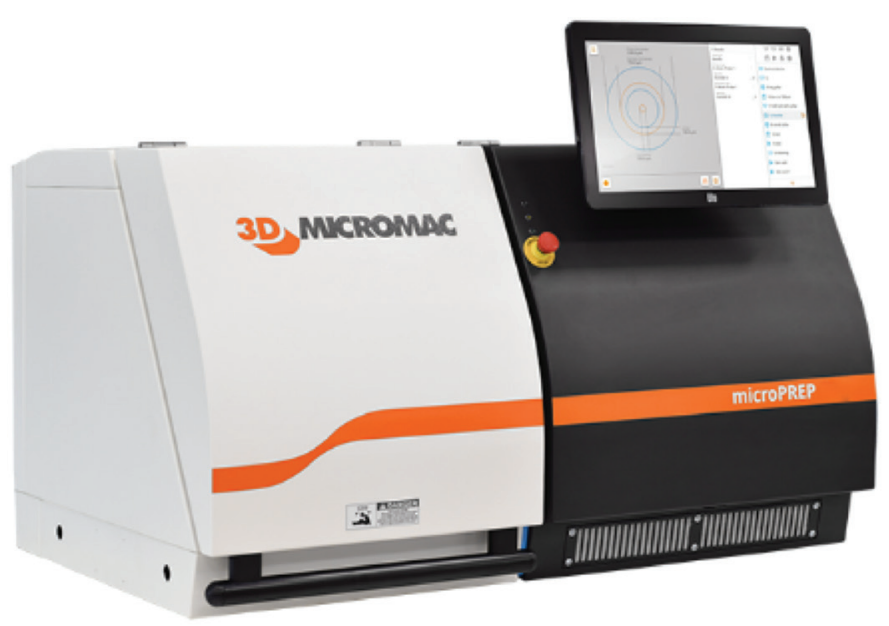

Figure 1: The 3D-Micromac microPREP, a laser-based specimen preparation system. 


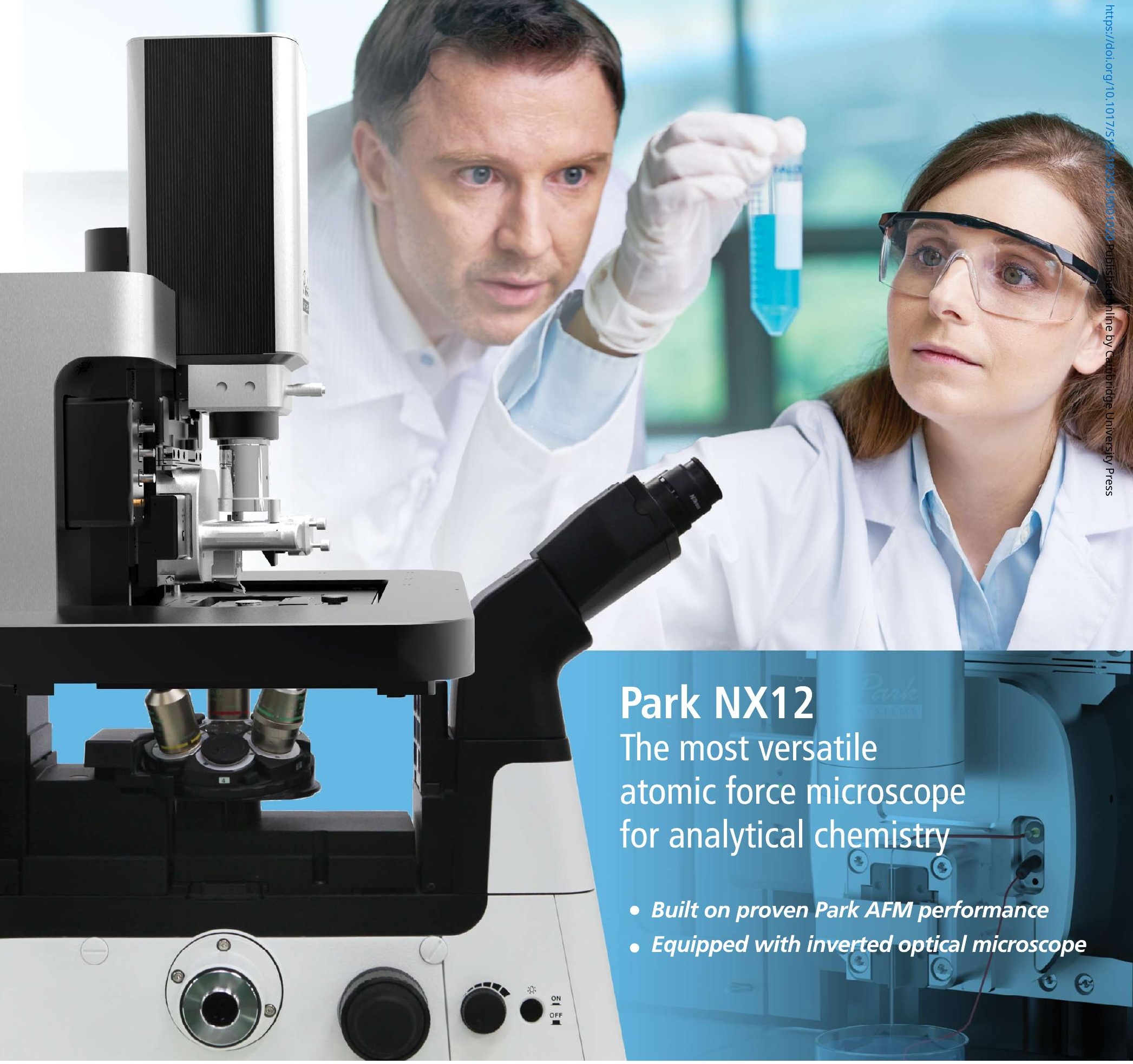

Proven Performance

The Park NX12 is based on the Park NX10, one of the most trusted and widely used AFMs for research. Users can rest assured that they are taking measurements with a cutting-edge tool.
Built for Versatility

Multi-user labs need a versatile microscope to meet a wide range of needs. The Park NX12 was built from the ground up to be a flexible modular platform to allow shared facilities to invest in a single AFM to perform any task.

\section{Competitive Pricing}

Early career researchers need to do great work with cost-effective tools. Despite its outstanding pedigree, the Park NX12 is priced affordably_-ideal for those on a constrained budget.

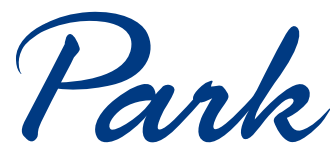


$5 \mathrm{~min}$

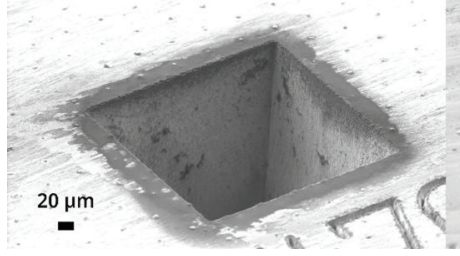

Figure 2: Boxmilling. (left) A Si sample $\left(300 \times 300 \times 250 \mu \mathrm{m}^{3}\right)$ machined by microPREP in 5 minutes. (right) Excavated through silicon via (TSV) in the box prepared by the microPREP and finished using a Zeiss Auriga 40 FIB.

a broad $\mathrm{Ar}^{+}$ion beam or the focused $\mathrm{Ga}^{+}$or $\mathrm{Xe}^{+}$ion beams used in FIB instruments. Figure 2 shows a $(300 \times 300 \times 250) \mu^{3}$ box milled by using the microPREP instrument. This specific preparation was done in less than $5 \mathrm{~min}$. If a FIB were used for the same task, it would take up to 17 days. Thus the removal of bulk material can be efficiently done by means of this laser instrument, but the speed still depends on the dimensions, the task, and the specific material to be removed.

Heat-affected zone. Widespread use of laser micromachining for the preparation of thin TEM specimens has been hampered by concerns regarding thermal damage to the sample. It is important to note that this effect can be controlled and minimized, depending on the material and the analysis task. The formation of a melt takes place when traditional pulsed laser (not ultra-short-pulsed) radiation interacts with matter and expels material from the location of machining. This heating is caused by the electromagnetic wave from the laser, which excites phonons and leads to a local increase in temperature.
For ultra-short pulsed lasers, however, the excitation is absorbed by the electronic system of the material, leading to much reduced heating effects. Ablation is not entirely "athermic," but it was found to be restricted to a depth of less than $0.4 \mu \mathrm{m}$ for silicon [6-9]. The extent of this heat-affected zone (HAZ) can become larger when basic principles of laser micromachining are not obeyed, for example, when the feed rate is too slow. Nevertheless, there is still a layer of material, micrometers in extent (worst case), which will sustain some structural damage. This structural damage strongly depends on the given material and the laser parameters used. To keep structural damage small and under control, the ablation process is restricted to a highly localized area. The HAZ is entirely removed by the FIB process. Depending on the material, the specific specimen, and the FIB parameters themselves, it can take few seconds to few minutes to remove the HAZ.

Workflows. Different workflows have been developed for processing bulk samples, cross sections, tomography, atomprobe pillars, and samples for micromechanical testing. Specimens for TEM and APT generally have starting dimensions of 1-3 mm. The workflow for cross-section preparation in the "XLChunk" mode is described below and shown in Figure 3.

The following cross-section workflow, based on the XLChunk approach, consists of five steps: (1) Laser milling of trenches/undercut, which will allow freeing the sample from the rest of the bulk material. (2) Laser cutting or milling of the required structures (H-bar, or pillars/tips). (3) Laser cutting of support structures. (4) Removal of workpiece and gluing to a copper half-ring. (5) Post-processing with an $\mathrm{Ar}^{+}$ion broad beam or with a focused $\mathrm{Ga}^{+}$or $\mathrm{Xe}^{+}$ion beam to remove structural damage in the HAZ.

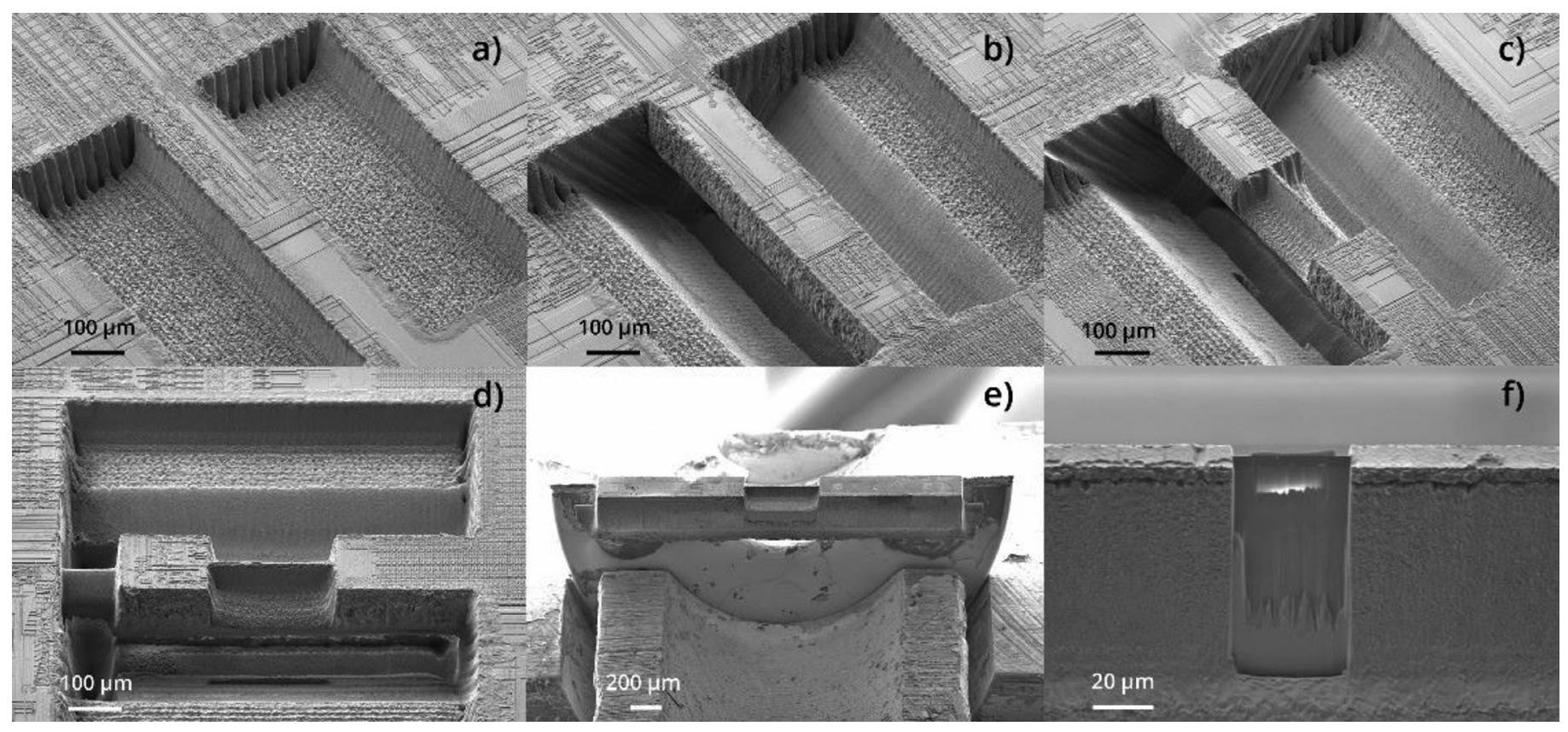

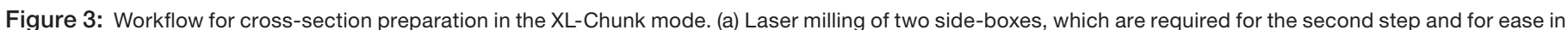

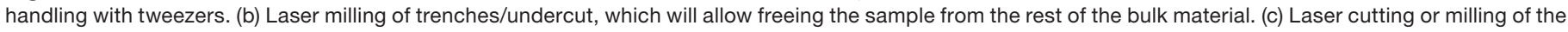

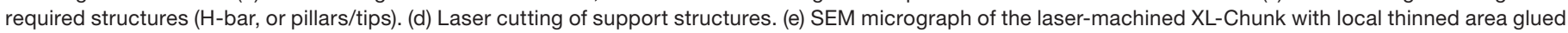

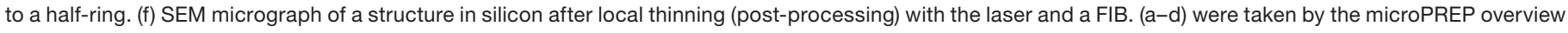
camera. (e-f) were taken with the SEM of a Zeiss Auriga 40. 

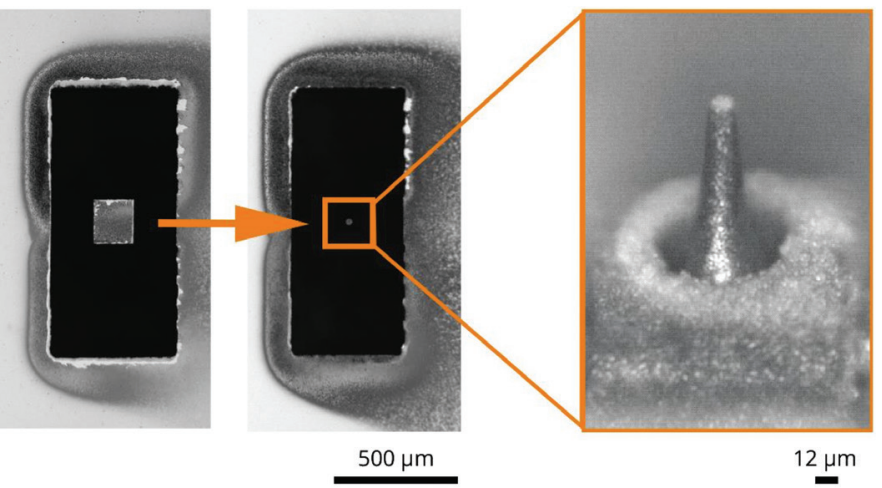

Figure 4: APT pillar preparation in silicon based on the XL-Chunk workflow. The pictures were taken by the microPREP overview camera.

This XL-Chunk workflow can be applied for TEM lamellae. Atom probe pillars can be prepared in two ways. One approach of a direct preparation of the pillar/tip on top of the XL-Chunk is shown in Figure 4. This illustrates that ultra-short-pulsed lasers are capable of fabricating tiny pillars in an automated fashion, which can be used as mounts or basic structures for APT.

Figure 5 shows a variety of forms and tasks that have been produced with this instrument for different methods of analysis. Another feature is the capability to upload CAD files. With this feature, regions of interest can be investigated by means of computer-aided navigation on the specimen, which is especially useful for semiconductor devices. Most tasks to be accomplished in this way involve quality assurance, failure analysis, or random sampling.

Cleaning. The aforementioned steps 1-5 for XL-Chunk preparation are done under ambient conditions without vacuum or inert gas atmosphere. Nevertheless, for the best results in terms of specimen cleanliness and an optimal start for post-processing steps, the instrument can be fitted with a contactless cleaning system called $\mathrm{CO}_{2}$ Snow Jet. This comprises a source of liquid $\mathrm{CO}_{2}$, a pressure reducer, a gas flow control unit, and a cleaning nozzle. After each processing step it is possible to add a cleaning step. The $\mathrm{CO}_{2}$ is inert to the specimen and can be applied without concerns regarding contamination or unwanted implantation effects. Figure 6 shows a specimen before and after snow jetting. The debris is broadly removed without interfering with the specimen.

Flexibility. The laser is a high-energy light with a final beam diameter of about $10 \mu \mathrm{m}$, which is directed by a precise laser scanner. The sample is held by a precise stage system (up to 6 axes), in order to position the region of interest in the center of the scan field. Thus, sample geometries like tips, bars, or other geometrical patterns can be machined from almost any type of material. Electron transparent sections can be made at one position or at multiple positions. In addition, multiple workflows allow the preparation of samples at several different, pre-determined positions, enabling automated high-throughput sample preparation. This mode is particularly valuable for metrology, evaluation of homogeneity, and screening applications.
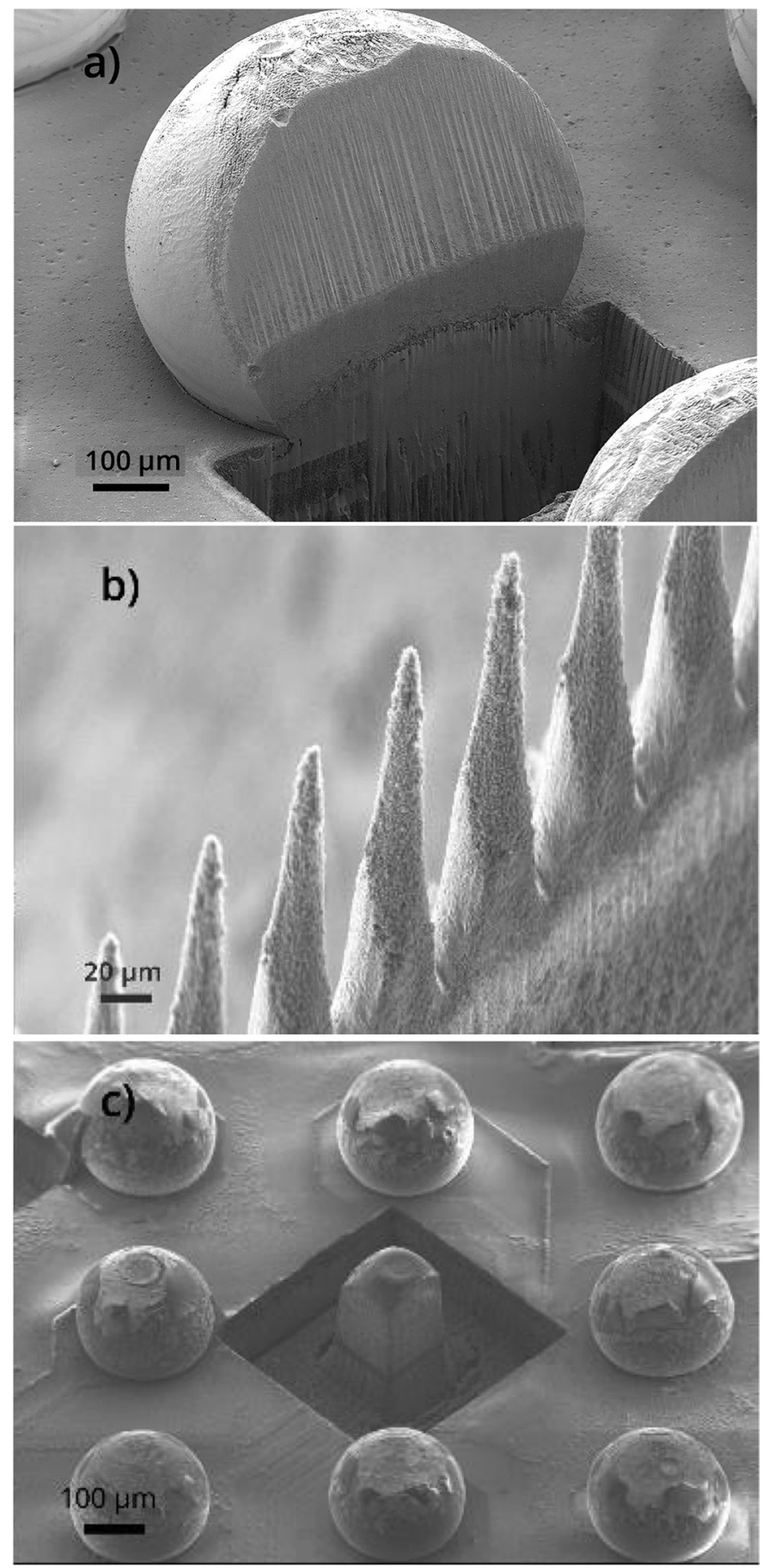

Figure 5: Examples of different preparation tasks showing the free form capability of the microPREP. (5a) Bump of lead-free solder SAC 305. (5b) Silicon APT samples. (5c) Ball grid array of lead-free solder SAC 305 bumps. SEM images taken with Carl Zeiss Supra55VP.

\section{Results}

Laser micromachining of H-bars in IC-structures. An $\mathrm{XL}$-chunk was prepared from an IC-structure while keeping supporting structures in place until the workpiece was removed with tweezers. In order to observe the structure in a TEM, the thinned region was glued to a $3 \mathrm{~mm}$ copper half-ring 

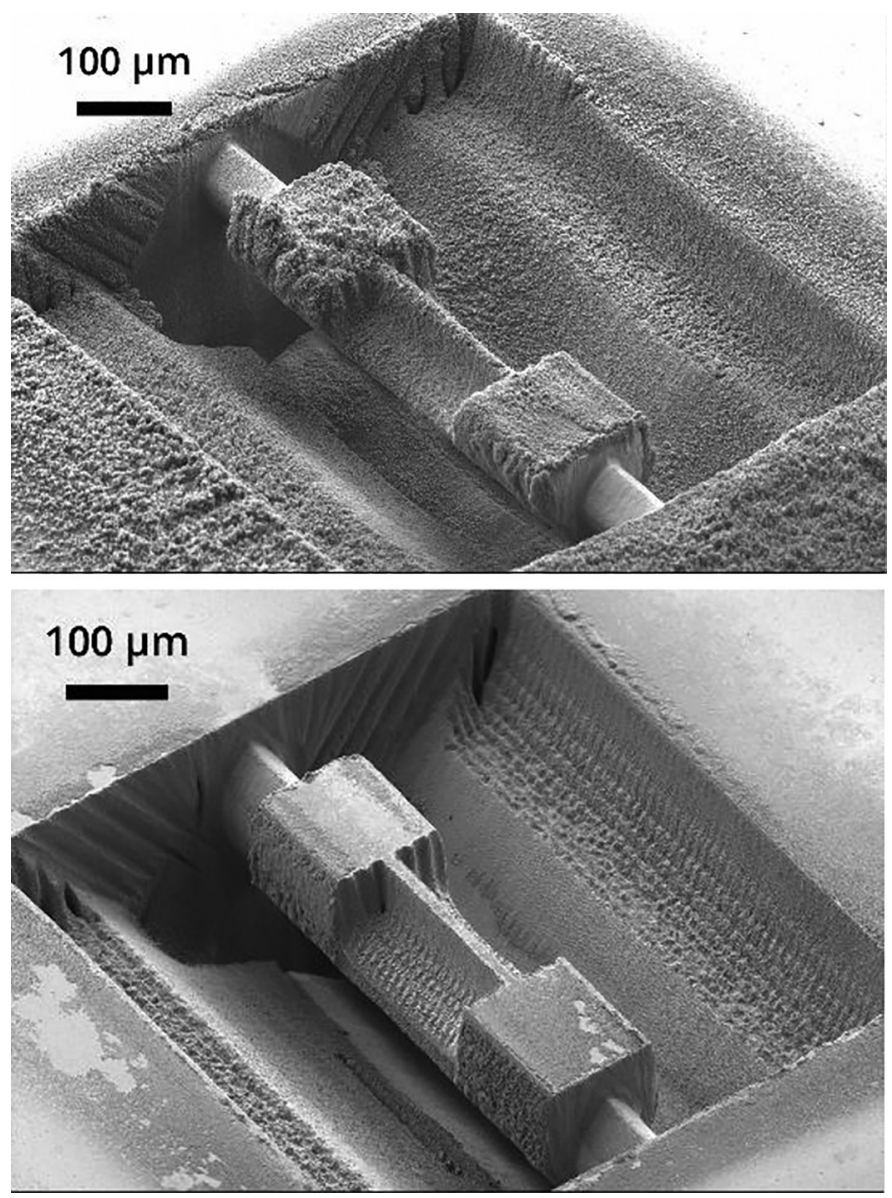

Figure 6: Specimen cleaning. (top) Specimen after laser processing without cleaning. (bottom) Specimen after processing and cleaning with $\mathrm{CO}_{2}$ Snow Jet. This specimen was machined by microPREP only, without additional FIB polishing.

(Figure 3e). In the next step, a FIB system was used to remove the HAZ and to thin the sample to the required thickness of $<100 \mathrm{~nm}$ (Figure 3f). The effectiveness of the instrument can be shown by milling a box $(300 \times 300 \times 250) \mu \mathrm{m}^{3}$ in silicon material within 5 minutes and the post-processing with a FIB (Figure 2). Figure 5 shows different preparation tasks and some free-form capabilities. Figure 6 shows the contactless nonabrasive cleaning using the $\mathrm{CO}_{2}$ Snow Jet.

\section{Discussion}

The XL-Chunk design is a support structure to hold TEM lamellae or atom probe tips. For APT, it is also possible to prepare several tips, as a line or an array of tips. It is also possible to mill complete APT cards. Modular design and flexible software allow the integration of this new type of chunk specimen prep into other workflows. Laser processing is an effective method for rapidly removing significant volumes of material to reduce the total time to a finished specimen. Since $\mathrm{CO}_{2}$ cleaning is nonabrasive, it is a useful process for removing redeposited material. Further developments are ongoing to reduce the HAZ and also to expose samples to a defined micro-environment (inert gas or vacuum) to minimize oxidation.

\section{Conclusions}

A new ultra-short-pulsed laser-based thin specimen preparation system provides fast, cost-effective, and site-specific preparation of samples for microstructure evaluation. The microPREP is not just a tool to prepare thin samples for TEM, it also can prepare specimens for APT and micromechanical testing. The modular stage and software design of the microPREP laser tool meets the exacting requirements of several industries.

\section{Acknowledgments}

The work presented in this paper has been performed in the project $\mathrm{SAM}^{3}$. The German partners are funded by the BMBF (German Federal Ministry of Education and Research) under contract 16ES0347, and the French partners are funded by the French Ministry for Industry and Economy. SAM ${ }^{3}$ is a joint project running in the European EUREKA EURIPIDES and CATRENE programs. We would like to thank all our partners in the aforementioned project.

\section{References}

[1] T Sato and J Byrnes, "TEM sample preparation workflow using laser ablation and broad ion beam milling," 19th International Microscopy Congress (IMC19), poster.

[2] J Byrnes et al., "A combined laser ablation/focused ion beam approach to atom probe sample preparation," 19th International Microscopy Congress (IMC19), poster.

[3] Schmidt, "Nanoscale 3D X-ray Microscopy for High Density Multi-Chip Packaging FA" $44^{\text {th }}$ International Symposium for Testing and Failure Analysis (ISTFA2018) (2018) $424-28$.

[4] Th Höche, PCT Patent Application WO 2013/026707 A1.

[5] M Krause and Th Höche, EP Patent Application EP 1316 2360.

[6] Th Höche et al., "Femto-second-Laser Interaction with Mo/Si Multilayer Stack at Low Fluence," Appl Phys A 79(4-6) (2004) 961-63.

[7] Th Höche et al., "Nanostructural Investigations on Ripples Prepared by Femto-Second Laser Treatment of Silicon," Proc. 2nd International Congress on Applications of Lasers \& Electro Optics (ICALEO), Jacksonville, FL, October 2003.

[8] D Ruthe et al., "Etching of CuInSe ${ }_{2}$ Thin Films - Comparison of Femtosecond and Picosecond Laser Ablation," Appl Surf Sci 247 (2005) 447-52.

[9] S Martens et al., "Simulation-Based Analysis of the HeatAffected Zone during Target Preparation by Pulsed-Laser Ablation through Stacked Silicon Dies in 3D Integrated System-in-Packages," $11^{\text {th }}$ Int. Conf. on Thermal, Mechanical and Multiphysics Simulation and Experiments in Micro-Electronics and Micro-Systems, EuroSimE, 2010. 


\section{The Capabilities in this Small Box are Beyond Your Imagination}

You don't have to imagine. It's real. Meet the Cube II, the world's smallest conventional SEM. At 143 lbs, it's a powerhouse in the industry today. Ease of use and the ability to look at just about any sample UNCOATED with no charging make this an unbelievable package worth seeing. Small in footprint but big on results, the Cube delivers what other SEMs cannot.

Images of uncoated glass wafer

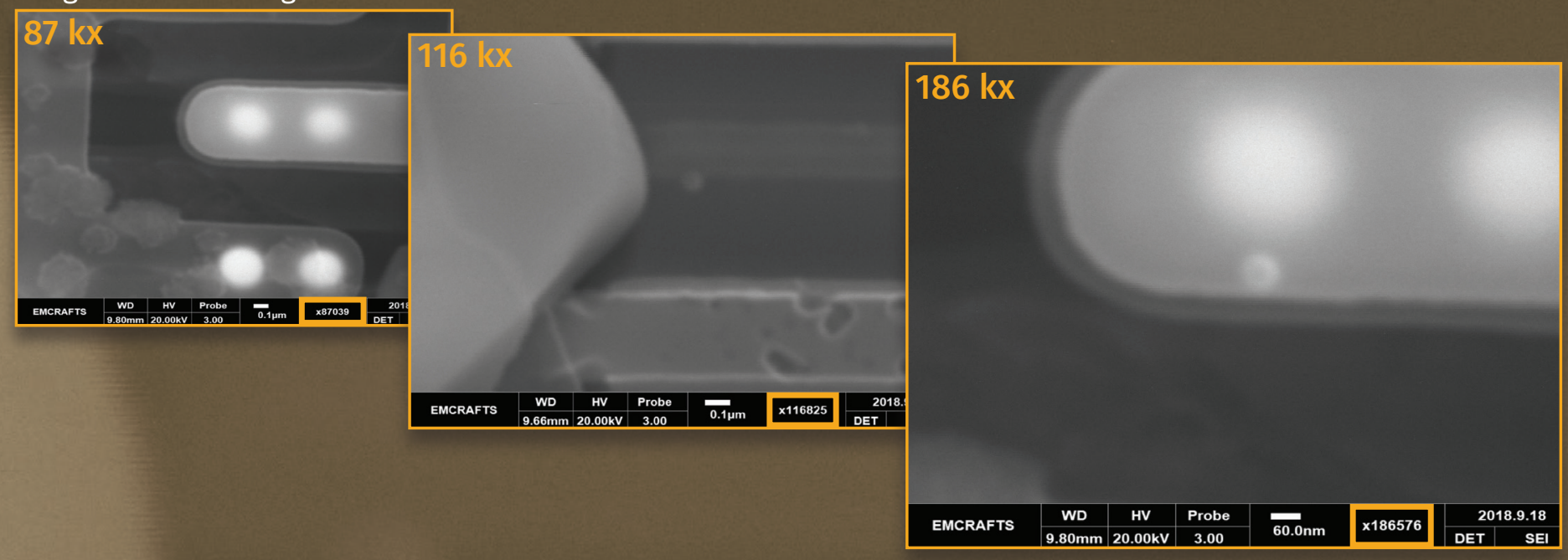

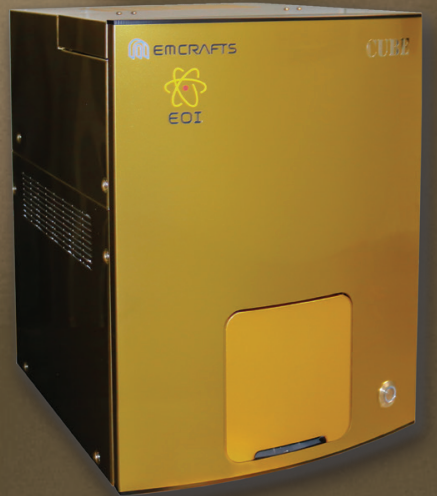

Cube II is only 17 in wide, 18 in deep and 21 inches tall to easily fit on a table or lab bench. -5.0nm SE image resolution on uncoated samples

-200-30kv acceleration voltage

- Magnification from $10 x$ to 300,000x

- Easy operation to shorten the ramp to high productivity

- Automated imaging mode

- Charging reduction mode

- Automated stage and 4-channel BSE

- Choice of integrated EDS options

\section{Don't just take our word for it! Bring us your sample!}

Try the Cube II for yourself in booth 1310 at MRS in Boston, December 3-5, to see the BIG RESULTS from this compact microscope. And while there, register to win an Apple Watch.*

*conditions apply
Electron Optics Instruments

Exclusive US Distributor of EmCrafts SEM

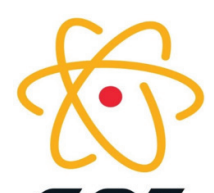

\section{EOI}

See our full line of SEMs or request more info: www.electronopticsinstruments.com info@electronopticsservice.com 


\section{Alphacen 300}

The only tip-scanning AFM for heavy samples and $300 \mathrm{~mm}$ wafers

New stage

New controller

New possibilities

At MRS?

Visit us at booth \#1018 to find out more. 